\begin{tabular}{l|l} 
REVISTA & $\begin{array}{l}\text { Revista Educación } \\
\text { ISSN: 0379-7082 } \\
\text { ISSN: 2215-2644 } \\
\text { revedu@ gmail.com } \\
\text { Universidad de Costa Rica } \\
\text { Costa Rica }\end{array}$
\end{tabular}

\title{
Comportamiento ético en la publicación científica: malas conductas y acciones para evitarlas
}

Zúñiga Vargas, Juan Pablo

Comportamiento ético en la publicación científica: malas conductas y acciones para evitarlas

Revista Educación, vol. 44, núm. 1, 2020

Universidad de Costa Rica, Costa Rica

Disponible en: http://www.redalyc.org/articulo.oa?id=44060092007

DOI: https://doi.org/10.15517/revedu.v44i1.35548

Esta obra está bajo una Licencia Creative Commons Atribución-NoComercial-SinDerivar 3.0 Internacional. 
Revisión bibliográfica

\title{
Comportamiento ético en la publicación científica: malas conductas y acciones para evitarlas
}

\section{Ethical Behavior in Scientific Publications: Misconduct and Preventive Actions}

Juan Pablo Zúniga Vargas

Universidad Nacional de Costa Rica, Costa Rica

juan.zuniga.vargas@una.ac.cr

(iD http://orcid.org/0000-0002-8717-3793

\author{
DOI: https://doi.org/10.15517/revedu.v44i1.35548 \\ Redalyc: http://www.redalyc.org/articulo.oa?id=44060092007
}

Recepción: 05 Mayo 2019

Aprobación: 18 Agosto 2019

\section{Resumen:}

El presente artículo desarrolla una revisión bibliográfica sobre las principales malas conductas científicas en torno a la publicación. Para este propósito, se ejecutó una búsqueda de información en bases de datos académicas y buscadores académicos en Internet para localizar publicaciones las cuales trataran el tema de la mala conducta científica en el proceso editorial de artículos científicos. La información localizada fue analizada y todo caso de mala conducta científica identificado fue clasificado de acuerdo con los participantes del proceso editorial de un artículo científico, a saber, personas autoras, editoras y dictaminadoras. A su vez, cada caso se discute concisamente. Finalmente, con el fin de luchar contra la mala conducta científica en la publicación, se sugiere recurrir a la evaluación formativa en los niveles de grado y posgrado (en cursos de metodología de la investigación, por ejemplo) y que los consejos editoriales de las revistas científicas adopten medidas para garantizar la ética en la publicación tales como códigos de ética y declaraciones de originalidad.

Palabras Clave: Fraude académico, Ética, Publicación, Científica, Investigación.

\section{Abstract:}

This article encompasses a bibliographic review of the main types of scientific misconduct in publications based on a search of academic databases and search engines. The search was performed publications regarding scientific editorial misconduct in research articles. The data obtained was analyzed and each example of misbehavior was classified according to author, editor and peer reviewers. For preventive purposes, formative assessments of undergraduate and graduate students understanding of research methodology are recommended. In addition, journal editorial boards must adopt measures, such as establish a code of ethics and statement of originality, to ensure ethical behavior when publishing research articles.

KEYwords: Academic Fraud, Ethics, Publication, Scientific, Research.

\section{INTRODUCCIÓN}

En todos los ámbitos de investigación, la principal fuente de literatura está constituida por artículos, los cuales son a su vez los más importantes medios de divulgación del quehacer académico e investigativo de las comunidades científicas a nivel mundial. Como tales, es fundamental que tanto en su desarrollo como en su proceso editorial se obedezcan serias normas éticas, puesto que la divulgación de información académica implica una gran responsabilidad en primera instancia con las comunidades mencionadas anteriormente, al ser las poblaciones que se benefician más inmediatamente de los hallazgos que se publican.

Asimismo, el público en general también tiene el derecho a acceder a información real y veraz. No obstante, en la actualidad existe una serie de circunstancias que pueden conducir a la mala conducta científica en la publicación. Por un lado, las tecnologías de la información y la comunicación (TIC) facilitan el compartir y recuperar artículos casi de manera inmediata, algo que era prácticamente impensable en los inicios de la publicación científica, cuando la comunicación de resultados de investigación ocurría por medio de unos pocos ejemplares de revistas publicados de manera física, los cuales eran leídos por muy pocas personas. 
Precisamente, esta misma inmediatez es la que en ocasiones hace pensar a las personas que toda la información habida en la Internet es de dominio público y que no existen ningún tipo de restricciones con respecto a su uso, lo cual puede verse traducido en casos de plagio cometidos con conocimiento de causa o sin él, solo por mencionar un ejemplo de mala conducta científica.

Por otra parte, las personas académicas universitarias se enfrentan a fuertes imposiciones del sistema. Para validar y ascender en sus carreras profesionales, deben publicar constantemente so pena de perder sus puestos o financiamiento para sus investigaciones (Tudela y Aznar, 2014). Esto causa a su vez que en esta condición de publicar o morir, se produzcan publicaciones cuya observancia de normas éticas pueda ser bastante cuestionable. Concretamente, la mala conducta científica es la principal causa de retractación de artículos (Campos y Ruano, en prensa).

No obstante, las personas autoras no son las únicas que enfrentan malas conductas en la publicación científica; las demás personas involucradas en el proceso editorial de las publicaciones académicas lidian también con desafíos éticos, los cuales se discutirán más adelante. La motivación principal para redactar el presente escrito nace de las inquietudes y retos enfrentados por quien escribe tanto en su investidura de editor de una revista científica como en su experiencia como tutor de trabajos finales de graduación a nivel de posgrado. Así pues, se procedió a ejecutar una búsqueda en bases de datos científicas (EBSCO Acedemic Search Complete y EBSCO Education and Research y ProQuest Central) y buscadores académicos en Internet (Google Académico, Bielefeld Academic Search Engine, Directory of Open Access Journals y ERIC), con el propósito de localizar publicaciones las cuales abordaran el tema de la mala conducta científica en la publicación y proceso editorial de artículos académicos.

Se revisaron las publicaciones encontradas y toda mala conducta identificada fue clasificada. Específicamente, la clasificación consideró a los tres principales actores involucrados en el desarrollo y revisión de una publicación académica de acuerdo con su cronología, a saber, las personas autoras de los escritos, las personas editoras de revistas científicas y las personas dictaminadoras de los artículos.

Es importante aclarar que en este artículo no se abordarán ningún tipo de malas conductas o consideraciones éticas relacionadas con la ejecución de investigaciones, pues su propósito principal será centrarse en el proceso de publicación per se de artículos científicos. Para cada grupo de clasificación, las malas conductas se consignan en orden alfabético para conveniencia de quien lee; no se desea, por lo tanto, establecer una jerarquía o categorización de cada caso según su seriedad. Finalmente, se espera que el presente manuscrito pueda ser de utilidad informativa y pedagógica para estudiantes universitarios en particular y para escritores académicos en general en el desarrollo de documentos de carácter científico.

\section{Principales malas conduCtas Científicas QUE ENFRENTAN LAS PERSONAS AUTORAS}

Como es de esperar, el mayor peso ético en la publicación científica yace sobre los hombros de las personas autoras. Así pues, son las personas autoras de artículos científicos las cuales deben lidiar con el mayor número de posibles malas conductas científicas en el desarrollo y publicación de artículos académicos. En este sentido, muchos pueden ser los motivos que lleven a una persona autora a cometer faltas de ética en la publicación de un artículo.

Por un lado, Buendía y Berrocal (2001) enumeran una serie de razones o presiones para efectuar publicaciones de ética dudosa:

1. competir para ofrecer primicias de investigación

2. obedecer a intereses comerciales para obtener financiamiento

3. conseguir un cierto nivel profesional

4. abusar de poder para ser publicado.

Por otro lado, Baiget (2010) menciona una lista más amplia de razones: 
1. presión por publicar o perecer

2. obtención de financiamiento

3. falta de integridad intelectual

4. ignorancia de la ética

5. promoción personal

6. arrogancia

7. fama

8. falta de conciencia moral

9. ganancias

10. deshonestidad

11. incompetencia

12. pereza

13. enfermedad mental.

Adicionalmente, Tudela y Aznar (2013) destacan tres causas principales para el fraude científico:

1. la búsqueda de reconocimiento

2. la presión para publicar en revistas científicas de alto factor de impacto

3. la primicia en divulgar un avance o descubrimiento.

Como se puede apreciar, las explicaciones o motivos para la falta de ética en la publicación científica son múltiples. Sin embargo, se describirán a continuación únicamente los casos más comunes de acuerdo con la indagación bibliográfica elaborada:

- Aumentar el número de referencias de un artículo (Santana, 2016): Con el afán de hacer parecer que se desarrolló una revisión de literatura exhaustiva, una persona autora podría inflar la lista de referencias de su artículo con fuentes de información no consultadas. Si el proceso de revisión y dictaminación de un artículo no es meticuloso en este sentido, se podría pasar por alto este aspecto aparentemente simple, pero de particular trascendencia en el desarrollo de un escrito académico.

- Cometer plagio en cualquiera de sus formas (Soto, 2012): Sin lugar a dudas, la comisión de plagio es la falta de ética más común y quizás más diversa en la publicación científica. Ya sea desde sus formas más burdas como copiar y pegar palabras ajenas sin ningún tipo de atribución hasta formas más sutiles como paráfrasis engañosas, el plagio ocurre generalmente al tomar información de otras personas sin el debido reconocimiento, aunque el autoplagio también suele ocurrir. De todas las malas conductas científicas en la publicación, esta es quizás la más sencilla de identificar, puesto que la existencia de diversos programas informáticos permite, con relativa facilidad, fragmentos de texto no originales.

- Eliminar injustificadamente un nombre (Dorta, 2013): Por diferentes motivos, aunque generalmente personales, se puede eliminar el nombre de una persona autora para que no figure en un artículo. En este sentido, las rencillas en equipos de investigación pueden suscitar esta mala conducta, la cual se puede ver facilitada, pues generalmente las revistas científicas solicitan que una de las personas autoras de un manuscrito sea designada como el contacto principal de correspondencia.

- Enviar una versión temprana de un artículo (Velásquez, 2014): Esta conducta poco ética ocurre cuando una persona autora envía un manuscrito a una revista a sabiendas de que no es publicable en su versión actual. Sin embargo, el envío se da como una manera gratuita de obtener realimentación para mejorar el artículo y publicarlo posteriormente, a costa del trabajo desperdiciado de la persona editora de la revista y de las personas que hayan leído el trabajo. Es posible argumentar además que las personas autoras que incurren en esta conducta probablemente tienen como objetivo el envío de un artículo a una revista de mayor prestigio, pero envían primeramente el manuscrito a una revista de menor prestigio, pero cuyos procesos de dictaminación de trabajos publicables son serios y críticos. 
- Falsificar o manipular datos de investigación (Anguiano, 2016): Esto ocurre cuando se parte de datos y metodologías reales de investigación. Sin embargo, la persona autora las modifica a conveniencia para que concuerden con sus objetivos o hipótesis de investigación. Llama la atención que se cometa esta mala conducta, pues en la investigación no siempre se encuentra lo que se busca. Sin embargo, en tanto se hayan seguido procedimientos adecuados en su desarrollo, los resultados negativos también pueden ser de interés. Por ejemplo, pueden mostrar qué no hacer o dar luz para nuevas rutas a seguir en una investigación.

- Incluir nombres falsos de personas coautoras (Biagioli, 2019): $\mathrm{Al}$ respecto de esta falta de ética se proponen tres motivaciones principales: 1 . si se efectúa una publicación fraudulenta, puede ser más seguro aparecer como parte de un equipo investigador y desviar la atención hacia una persona específica, 2. si el desarrollo de una investigación requiere de destrezas o atestados que la persona autora no posee, se incluyen a personas coautoras falsas que sí los posean, y 3. si la persona autora no es muy conocida en la comunidad científica, se pueden utilizar nombres falsos vinculados a instituciones reales para dar mayor peso y credibilidad a la publicación. En resumidas cuentas, da más seguridad estar dentro de un grupo que estar solo incluso en las malas conductas científicas.

- Incluir una autoría de regalo (Anguiano, 2016; Lam, 2018): Esta es una falta de ética que ocurre cuando se incluye el nombre de una persona como coautora de un artículo, aunque no haya contribuido de manera alguna en su desarrollo. Puede darse como pago por algún favor recibido. En sí, se puede ver como una forma más de traficar influencias en la academia.

- Incluir un nombre por coerción o compromiso (Hernández, 2007): Cuando una persona ostenta autoridad sobre otras puede exigir que su nombre sea incluido en un artículo, aunque no haya contribuido a este. También puede suceder que la inclusión de tal nombre sea algo así como un compromiso adquirido. Estos casos pueden ocurrir con personas en puestos de jefatura, coordinación o docencia hacia las personas subalternas, supervisadas o estudiantes. Cuando una persona ostenta autoridad sobre otra, dicha autoridad puede verse comprometida moralmente, aunque un buen liderazgo debería estar ajeno a tales abusos.

- Intercambiar citas con otras personas autoras (Vázquez, 2016): Esta falta de ética ocurre cuando dos o más personas autoras convienen a citarse las unas a las otras en sus trabajos para aumentar el índice de citación de sus publicaciones. Nuevamente se puede estar ante una manifestación del tráfico de influencias en la academia.

- Inventar datos de investigación (Anguiano, 2016; Lam, 2018): Esto sucede cuando se generan datos de investigación parcial o totalmente sin ningún proceso científico de por medio. De acuerdo con Buendía y Berrocal (2001), dos razones para el uso de datos falsos en investigaciones son: 1. confirmar hipótesis y 2. conseguir mayor reputación. Puede argumentarse además que esto podría resultar más o menos difícil de lograr según el paradigma de investigación que se siga y el tipo de investigación que se desarrolle.

- Modificar la composición de las personas autoras (Mayta y Curioso, 2009): Con el ánimo de hacer parecer que un artículo es nuevo, se puede cambiar el orden o cantidad de las personas autoras y someterlo nuevamente a otra revista. En el caso de una revista depredadora, será muy sencillo que el artículo sea publicado.

- Modificar la designación de la persona autora principal (Vázquez, 2016): Con el afán de obtener más prestigio, se puede llegar a modificar el orden de aparición de las personas autoras en un artículo y designar como autora principal a la persona que más convenga, con o sin conocimiento de las demás personas coautoras. Como se mencionó anteriormente, se facilita hacer esto cuando se designa a una sola persona como contacto principal de correspondencia.

- No declarar conflictos de intereses ${ }^{[1]}$ : Toda persona autora de un artículo científico debe declarar cualquier conflicto de intereses que pudiera existir con la publicación. En especial, las fuentes de 
financiamiento para una investigación podrían ejercer una influencia sobre los resultados que se reporten. Dicho de otro modo, se pueden llegar a reportar únicamente los resultados que sean convenientes para la entidad que financia el estudio.

- Efectuar autocitas excesivas (Vázquez, 2016): Este dilema ético tiene lugar cuando una persona autora cita sus trabajos de manera desmesurada con el propósito de aumentar el índice de citación de sus publicaciones. Por este motivo, los índices de impacto de artículos científicos deben ser analizados cuidadosamente. No todas las citas recibidas son legitimas.

- Realizar cambios al título de un artículo (Mayta y Curioso, 2009): Una estrategia deshonesta para hacer parecer a un artículo como una publicación completamente nueva es hacerle cambios en el título para someterlo a otra revista. Por este motivo, siempre es pertinente indagar todos los manuscritos que son recibidos en una revista, ya sea por medio de programas para la detección de plagio o en su defecto por medio de búsquedas en Internet.

- Realizar cambios en la bibliografía de un artículo (Mayta y Curioso, 2009): Aumentar o actualizar la bibliografía de un artículo puede ser una estrategia para hacer creer que un artículo es nuevo o distinto a lo ya publicado y presentarlo a otra revista. El acervo de publicaciones crece constantemente, por lo que se suele creer que únicamente con la inclusión de referencias más recientes se está produciendo un artículo nuevo.

- Efectuar envíos simultáneos de un mismo manuscrito (Vázquez, 2016): Esta es una falta a la ética muy común, en la cual una persona autora realiza el envío de un mismo manuscrito a más de una revista con la esperanza de que sea publicado en la primera revista que lo acepte. En el peor de los casos, el mismo manuscrito podría ser publicado por más de una revista.

- Realizar publicaciones infladas (Bravo, 2000): Este tipo de falta de ética, también conocido como meat extenderpublication en inglés, ocurre cuando se publica o se somete para publicación un artículo que ya ha sido publicado y únicamente se le añade información o datos adicionales en sus diferentes secciones. Así pues, el hecho de que se amplíe uno o más apartados de un artículo no significa que se ha generado un manuscrito completamente distinto.

- Ejecutar publicaciones segmentadas (Vázquez, 2016): Conocida también como publicación salami o salami slicing (por su denominación en inglés), esta falta a la ética ocurre cuando una persona autora divide un estudio o investigación en diferentes partes y trata de hacerlas pasar como trabajos diferentes. No debe confundirse, sin embargo, con las diferentes etapas de un proyecto de investigación a través de los años que se puedan ver sistematizadas por medio de publicaciones.

- Usar personas autoras fantasmas: Esta falta de ética se ha descrito en el contexto de las ciencias médicas, en las cuales compañías farmacéuticas, para propósitos de mercadeo, "[c]ontratan a personal específico especializado para escribir los artículos médicos e intentan que dichos artículos los firmen investigadores de prestigio que habitualmente no han hecho ninguna contribución sustancial al artículo" (Avanzas, Bayes, Pérez de la Isla, Sanchis y Heras, 2011, p. 428) y las personas que originalmente redactaron el artículo no figurarán como autoras. No está de más considerar que aquí habría dinero de por medio, sin mencionar la veracidad de la información que se consigne en el artículo.

Principales malas conductas científicas que enfrentan las Personas editoras de REVISTAS CIENTÍFICAS

Las personas editoras desempeñan una labor crucial en el buen funcionamiento de las revistas científicas y tal investidura viene acompañada de desafíos para la ética: 
- Imponer citas (Vázquez, 2016): Esta falta a la ética puede suceder si la persona editora de una revista obliga a una persona autora a incluir referencias a artículos de su revista, con el fin de aumentar el factor de impacto de dicha revista. Sin embargo, para desarrollar antecedentes y construir marcos teóricos se debe recurrir a todas las publicaciones que existan sobre un tema determinado, no de una revista en específico, ni mucho menos por medio de la coerción.

- Imponer un sesgo de publicación (Vázquez, 2016): La persona editora de una revista puede decidir deliberadamente solo aceptar publicaciones relacionadas con temas de su preferencia o conveniencia. Más allá del enfoque y alcance que se espera que tenga toda revista, se podría llegar al extremo de imponer un sesgo personal con respecto a cuáles artículos sean aceptados y publicados. Por otro lado, de acuerdo con Lam (2018) "los estudios con resultados positivos o estadísticamente significativos se publican más que los que tienen resultados negativos o estadísticamente no significativos" (p. 99); no obstante, es importante señalar que el desarrollo de la ciencia y el conocimiento humano debe nutrirse tanto de los resultados positivos como de los negativos. De ambos casos es posible aprender mucho, como ya se mencionó anteriormente.

- Modificar las citaciones en artículos aceptados (Pérez y Amaya, 2017): Una persona editora con pocos escrúpulos podría efectuar cambios en las citaciones de un artículo que ya ha sido aceptado con el fin de incluir artículos de su revista con miras a aumentar el factor de impacto de dicha revista, pero se debe recordar siempre que, en su investidura, una persona editora se debe a la comunidad, no al beneficio inescrupuloso de su propio proyecto editorial.

- No contactar a personas dictaminadoras idóneas: La emisión de un juicio válido y objetivo con respecto a un artículo publicable dependerá en gran medida de que la persona editora de una revista científica contacte a personas dictaminadoras con un perfil y conocimiento académicos idóneos para elaborar la revisión. El gran reto aquí yace en la especificidad de las temáticas de los manuscritos que se estén revisando, pues hay campos de investigación muy específicos cuyo número de especialistas es reducido.

- No declarar conflictos de intereses ${ }^{[2]}$ : Toda persona editora de una revista científica debe declarar cualquier conflicto de intereses que pudiera existir con el proceso editorial de un artículo publicable. De existir alguno, la persona editora está en la obligación de delegar su función a otra persona integrante del consejo editorial de la revista.

- No velar por la confidencialidad en los procesos editoriales (Tur, Fonseca y Gutiérrez, 2012): Como parte de un proceso de dictaminación objetivo, las identidades de las personas autoras y de las personas dictaminadoras deben permanecer en el anonimato. De otro modo, se podrían generar problemas éticos. Por ejemplo, si las personas autoras y dictaminadoras se conocen, se podría emitir un dictamen subjetivo y mediado por un conflicto de intereses.

- Utilizar información proveniente de un artículo científico en revisión (Velásquez, 2010): Una persona editora de una revista científica asume una serie de compromisos a la hora de recibir un trabajo publicable. Dentro de estos compromisos, un dilema ético sería utilizar información de un trabajo que se encuentre en proceso editorial en una de sus investigaciones. En tanto no sea publicado oficialmente el artículo, la persona editora de la revista o cualquier persona integrante del consejo editorial debería abstenerse de utilizar cualquier tipo de información proveniente del artículo. Sin embargo, si la revista permite que las personas autoras archiven la versión pre-print de los artículos en repositorios o plataformas similares, esto no debería ser un problema en tanto se utilice una referencia adecuada al documento en cuestión. 


\section{Principales malas conductas científicas QUE enfrentan las personas DICTAMINADORAS DE ARTÍCULOS}

La mayoría de las revistas logran salir adelante con los procesos editoriales de los artículos que reciben gracias a la colaboración, generalmente ad honorem, de un cuerpo de personas dictaminadoras, quienes también se enfrentan a ciertos dilemas éticos en su labor:

- No declarar conflictos de intereses ${ }^{[3]}$ : Toda persona dictaminadora debe declarar cualquier conflicto de intereses que pudiera existir y que pueda afectar la emisión de un juicio objetivo para un artículo publicable. Como se dijo anteriormente, un conflicto de intereses no declarado puede influenciar positiva o negativamente en la emisión de un dictamen.

- Realizar revisiones inadecuadas (Vázquez, 2016): Ya sea por negligencia, desinterés o incompetencia académica, una persona dictaminadora podría efectuar una revisión inadecuada, la cual podría afectar la publicación o no de un artículo. A criterio de quien escribe, no convienen los extremos en los procesos de dictaminación de artículos publicables. No es pertinente aceptar todo manuscrito sin ningún tipo de filtro, pero tampoco se deberían rechazar artículos que tengan el potencial de ser mejores. Para poder dictaminar un artículo, se requiere no solo conocimiento disciplinar, si no también conocimiento sobre cómo revisar un manuscrito académico con la mayor objetividad posible. Aquí también debe intervenir el buen juicio de la persona editora de una revista científica.

- Utilizar información proveniente de un artículo científico en revisión (Velásquez, 2010): Por más útil que pueda ser la información plasmada en un artículo en revisión, una persona dictaminadora debe abstenerse de utilizarla en sus investigaciones hasta tanto no se publique oficialmente el artículo. Ya se indicó que si la versión pre-print está disponible en algún servicio de almacenamiento en Internet, esto no debería ser un problema.

\section{ConcLuSIONES}

En el presente artículo, se han mencionado las principales razones por las cuales se pueden dar casos de mala conducta científica en la publicación. Para mayor conveniencia de quien lee, cada caso se clasificó de acuerdo con su ámbito de ocurrencia más común en el proceso editorial de un manuscrito publicable. Se espera que este trabajo sea esclarecedor en materia ética en la escritura académica y pueda ser de provecho para personas docentes y estudiantes principalmente en el contexto de la educación superior. Seguidamente, se considerarán opciones generales para lidiar con las malas conductas descritas en este artículo.

Pensar en soluciones absolutas o inmediatas para velar por la ética en la publicación científica es poco factible. Así pues, utilizar sanciones tales como cartas de amonestación o el retiro de circulación de un artículo (Elli, 2015) como estrategias disuasivas para la comisión de faltas a la ética son maneras de abordar el problema, pero a criterio de quien escribe no lograría cambios significativos en la conducta científica. Por ejemplo, França (2014) indica que existen complicaciones de orden personal y organizacional a la hora de denunciar una mala conducta científica. En el plano personal, quien denuncia se expone a ser señalado y acosado por ser un colega desleal. En el plano organizacional, una persona denunciante puede exponerse a sanciones y amonestaciones, puesto que " $\mathrm{s}$ ] e le 'cree' al investigador denunciado más que al denunciante bien fundado en sus datos. Pareciera que el investigador ya establecido, como tal, adquiere una especie de 'aureola de santidad' imperturbable e impenetrable” (França, 2014, p. 97).

Por lo tanto, lo más pertinente es pensar en diferentes medidas (principalmente formativas) orientadas a la promoción de la ética en la publicación científica. Esto para nada es una tarea sencilla y debe ser visto como 
un proceso con diferentes ámbitos de acción. $\mathrm{Al}$ menos las siguientes dos medidas deberían ser tomadas en cuenta en búsqueda de una ética más sólida en la publicación científica.

Como primer ámbito de acción, el principal pilar para sustentar la ética en la publicación científica debe estar en la educación. En todo contexto educativo (pero especialmente en la educación superior), la ética debe ser un eje transversal y esta temática debe abordarse recurrentemente y con ejemplos concretos para que quede claro qué se debe entender por ética o buena conducta en la redacción y publicación de artículos científicos o manuscritos académicos. En sí, este no es un tema cuyo abordaje sea simple, pues como señala Velásquez (2010), "el comportamiento ético pareciera aprenderse más por el contacto con los tutores y docentes, que por dictarse como un tema regular en las asignaturas de posgrado" (p. 7). Por lo tanto, el profesorado universitario debe ver en la evaluación formativa un recurso idóneo para orientar a sus estudiantes hacia la observancia de normas éticas en la escritura académica. Dicho cometido puede lograrse por medio de la práctica continua y con un abordaje no punitivo en todo momento en el que se requiera la escritura académica, por ejemplo, en un curso de metodología de la investigación. A diferencia de lo expuesto por Velásquez (2010), la ética debe abordarse explícitamente desde el nivel de pregrado, no solo desde el de posgrado.

Como segundo ámbito de acción, es de suma importancia que las revistas científicas adopten prácticas tendientes a promover la ética. Los consejos editoriales de estas deben adoptar o desarrollar códigos de ética y buenas prácticas, los cuales deben ser conocidos y suscritos por todas las personas involucradas en los procesos editoriales de los artículos científicos. La selección de personas dictaminadoras idóneas debe ser un proceso constante y crítico para identificar a aquellas con los mejores perfiles y juicios. En relación con este punto, las revistas científicas deben desarrollar normas de publicación claras e instrumentos de valoración de trabajos publicables que ayuden a las personas dictaminadoras a emitir dictámenes válidos y objetivos.

Es recomendable que las revistas generen declaraciones en las cuales queden claro los compromisos que las personas autoras adquieren al hacer un envío, tales como el someter manuscritos originales, no efectuar envíos simultáneos y declarar cualquier conflicto de intereses que pudiese existir y que deban acompañar a cada artículo sometido para publicación. Es deseable además que las revistas científicas utilicen programas informáticos especializados en la detección de plagio para revisar todos los artículos que reciben y tomar las acciones necesarias con respecto a los resultados obtenidos por medio de estos recursos tecnológicos.

Finalmente y, en todo caso, las personas integrantes de los consejos editoriales de las revistas científicas deben contar con los recursos necesarios que faciliten sus labores. En especial, la capacitación constante con miras hacia la profesionalización en la gestión de revistas científicas. Por lo tanto, el apoyo institucional será crucial en la promoción de buenas conductas en la publicación científica.

\section{REFERENCIAS}

Anguiano, J. (2016). Ética de la publicación científica: análisis y discusión filosófica sobre los principios del acto de publicar en las ciencias. Avances en Investigación Agropecuaria, 20(1), 5-14. Recuperado de https://bit.ly/32sM bj0

Avanzas, P., Bayes, A., Pérez de la Isla, L., Sanchis, J. y Heras, M. (2011). Consideraciones éticas de la publicación de artículos científicos. Rev Esp Cardiol, 64(5), 427-429. doi: https://doi.org/10.1016/j.recesp.2011.02.006

Baiget, T. (2010). Ética en revistas científicas. Ibersid: Revista de Sistemas de Información y Documentación, 4, 59-65. Recuperado de https://bit.ly/2JEmbd1

Biagioli, M. (2019). “A new form of plagiarism:” When researchers fake co-authors' name. Retraction Watch. Recuperado de https://bit.ly/2XLWgsM

Bravo, R. (2000). Aspectos éticos en las publicaciones cientificas. Recuperado de https://bit.ly/2JGSE2I

Buendía, L. y Berrocal, E. (2001). Ética de la investigación educativa. Agora Digital, 1, 1-14. Recuperado de https:// bit.ly/2hecmD1 
Campos, I. y Ruano, A. (2019). Misconduct as the main cause for retraction. Gaceta Sanitaria. doi: https://doi.org/ 10.1016/j.gaceta.2018.01.009

Dorta, A. J. (2013). Ética de la publicación científica. Revista Habanera de Ciencias Médicas, 12(4), 487-491. Recuperado de http://scielo.sld.cu/pdf/rhcm/v12n4/rhcm01413.pdf

Elli, J. R. (2015). Ética de la comunicación científica. Neurología Argentina, 7(1), 59-62. Recuperado de https://bit. ly/2YV9oZ1

França, O. (2014). Estrategias para inhibir y prevenir el fraude en la investigación científica. Revista Latinoamericana de Bioética, 14(2), 90-99. Recuperado de http://www.scielo.org.co/pdf/rlb/v14n2/v14n2a08.pdf

Hernández, F. (2007). Fraude en la autoría de artículos científicos. Rev Biomed, 18(2), 127-140. Recuperado de htt ps://bit.ly/2SfHPHn

Lam, R. M. (2018). Mala conducta científica en la publicación. Revista Cubana de Hematología, Inmunología y Hemoterapia, 34(1), 96-101. Recuperado de http://scielo.sld.cu/pdf/hih/v34n1/a12_546.pdf

Mayta, P. y Curioso, W. H. (2009). Política editorial ante la detección de una publicación redundante. Rev Peru Med Exp Salud Pública, 26(1), 5-8. Recuperado de http://www.scielo.org.pe/pdf/rins/v26n1/a02v26n1.pdf

Pérez, A. M. y Amaya, L. (2017). Retos contemporáneos a la ética en el proceso de la publicación científica. Avances en Psicologia Latinoamericana, 35(3), 427-431. Recuperado de https://bit.ly/2JAuZAU

Santana, L. (2016). Principales problemas éticos que se manifiestan en el proceso depublicación. Ponencia presentada en el Congreso Internacional de Información INFO 2016. Instituto de Información Científica y Tecnológica, Cuba.

Soto, A. (2012). El plagio y su impacto a nivel académico y profesional. E-Ciencias de la Información, 2(1), 1-13. Recuperado de https://bit.ly/2hIn4Cp

Tudela, J. y Aznar, J. (2014). El fraude en la publicación científica: una polémica que no cesa. Persona y Bioética, 18(2), 153-157. Recuperado de https://bit.ly/2JA82NW

Tudela, J. y Aznar, J. (2013). ¿Publicar o morir? El fraude en la investigación y las publicaciones científicas. Persona y Bioética, 17(1), 12-27. Recuperado de https://bit.ly/1MqeiyW

Tur, V., Fonseca, M. C. y Gutiérrez, B. (2012). Ética de la publicación científica: iniciativas y recomendaciones. El Profesional de la Información, 21(5), 491-497. Recuperado de https://bit.ly/2XRgktT

Vázquez, S. E. (2016). Ética en la publicación de revistas académicas: percepción de los editores en ciencias sociales. Innovación Educativa, 16(72), 53-74. Recuperado de https://bit.ly/2JOOGnr

Velásquez, J. D. (2014). Consejos para evitar problemas éticos en la publicación científica. Dyna, 81(187), 11-20. Recuperado de https://bit.ly/2LuEHqo

Velásquez, J. D. (2010). Sobre la ética en la publicación científica. Revista Avances en Sistemas e Informática, 7(3), 7-10. Recuperado de https://bit.ly/2YXV9m5

\section{Notas}

[1] De acuerdo con Vázquez (2016), “[d]ichos conflictos pueden ser académicos, personales, económicos, políticos o comerciales" (p. 62).

[2] De acuerdo con Vázquez (2016), "[d]ichos conflictos pueden ser académicos, personales, económicos, políticos o comerciales" (p. 62).

[3] De acuerdo con Vázquez (2016), "[d]ichos conflictos pueden ser académicos, personales, económicos, políticos o comerciales" (p. 62).

\section{BY-NC-ND}

